\title{
Multiple symmetric lipomatosis with vulvar involvement: a rare case report associated with walking difficulty and urination disorder
}

\author{
Maiko Kiyama $^{1} \cdot$ Koji Kanayama $^{1}$ (D) Solji Roh ${ }^{1} \cdot$ Yoshitsugu Hattori $^{1} \cdot$ Jun Oba ${ }^{1} \cdot$ Takuya lida $^{1} \cdot$ Tetsuo Ushiku $^{2}$. \\ Mutsumi Okazaki ${ }^{1}$
}

Received: 21 April 2019 / Accepted: 28 August 2019 / Published online: 4 September 2019

(C) The Author(s) 2019

\begin{abstract}
Multiple symmetric lipomatosis (MSL) - a rare metabolic condition characterized by diffuse, nonencapsulated, painless fat deposits - remains a challenging condition to treat. In addition, the clinical manifestation of MSL in women has not been fully understood due to the male-dominated prevalence of the disease. Here, we report a rare case of MSL with vulvar involvement and provide a review of the literature to increase the understanding of both the diagnosis and treatment of the disease. A 46-year-old Japanese woman, who had a history of chronic alcohol consumption for over 20 years, presented with bilateral giant enlargement of the labia major and complained of difficulty in walking and urination. Magnetic resonance imaging revealed nonencapsulated fat deposits involving the vulvar region and extending around the rectum. The patient underwent pubic lipectomy followed by episioperineoplasty, which resulted in a good esthetic outcome with sufficient patient satisfaction. The morphological presentation of MSL in women is different from that in men, and clinical expression of MSL in the vulvar region is rare but cannot be overlooked owing to the potential of serious sequelae such as walking difficulty, urination disorder, and sexual dysfunction. In such cases, lipectomy followed by episioperineoplasty is indicated for the treatment of the condition and its sequelae, while ensuring less chance of relapse and better esthetic outcome compared with other treatment strategies.

Level of evidence: Level V, therapeutic, diagnostic, risk/prognostic study.
\end{abstract}

Keywords Multiple symmetric lipomatosis $\cdot$ Female $\cdot$ Lipectomy $\cdot$ Alcoholism $\cdot$ Vulva

\section{Introduction}

Multiple symmetric lipomatosis (MSL), also known as Madelung disease, is a rare condition characterized by an accumulation of multiple nonencapsulated lipomatous tissues with a symmetric distribution that was first reported by Brodie [1], Madelung [2], and Launois [3]. The characteristic metabolic pattern of MSL is well described in men, including the very characteristic "buffalo hump" or "horse collar (Madelung collar)." However, the clinical expression of MSL in women is different from that in men; the typical morphologic presentation in women is characterized by fat

Koji Kanayama

kanayama-tky@umin.ac.jp

1 Department of Plastic and Reconstructive Surgery, The University of Tokyo, 7-3-1 Hongo, Bunkyo-ku, Tokyo 113-8655, Japan

2 Department of Pathology, The University of Tokyo, 7-3-1 Hongo, Bunkyo-ku, Tokyo 113-8655, Japan deposition in the proximal arms and legs, giving the patient a "football player" appearance [4]. Thus, such sex-related morphologic features could lead to the underdiagnosis of the disease in women.

There have been few reports of MSL involving external genital organs that play a role in the process of human reproduction. In men, only 8 cases of MSL affecting the scrotal region have been reported [5]. In women, MSL with vulvar involvement affecting the mons pubis was first described by Tan et al. [6] in 2008 and remains such a rare condition that no other cases have been reported in the English literature to date. The distribution of lipomatosis in the perineal region is not only rare but attracts clinical interest because it may create problems such as sexual dysfunction, urination disorders, and difficulty in walking. Here, we report a new case with vulvar involvement, which is also the first case of MSL affecting the labia majora, with a review of the literature to increase the understanding of both the diagnosis and treatment of the disease. 


\section{Case report}

A 46-year-old Japanese woman presented with a 3-year history of painless, soft, slow-growing swelling of the labia majora, which was causing difficulty in walking and urination (Fig. 1). She had a history of drinking both $1000 \mathrm{~mL}$ of beer (5\% alcohol content) and $180 \mathrm{~mL}$ of spirits (20-25\% alcohol content) at least every day for over 20 years, resulting in decompensated liver cirrhosis. Physical examination revealed bilateral giant masses affecting the labia majora, cheek, and upper arm, whereas there were no masses on the neck or shoulders. Magnetic resonance imaging (MRI) revealed nonencapsulated fat deposits in the labia majora and extending around the rectum (Fig. 2). Computed tomography (CT) revealed nonenhancement of the accumulated fat and no apparent enlargement of the inguinal or pelvic lymph nodes. Laboratory data showed both hyperbilirubinemia and hypoalbuminemia with thrombocytopenia. Excisional biopsy was performed, and the pathologic tissue demonstrated diffuse overgrowth of mature adipocytes, suggesting no malignancy.

The patient underwent pubic lipectomy followed by episioperineoplasty (Fig. 3). The lipomatous tissue lacked encapsulation and the transition between lipomatous and normal tissue was poorly demarcated. Two specimens, measuring $20 \times 17 \times$ $6 \mathrm{~cm}$ (weighing $900 \mathrm{~g}$ ) in the left and $17 \times 12 \times 7 \mathrm{~cm}$ (weighing $640 \mathrm{~g}$ ) in the right labia majora, were removed and subsequent esthetic reconstruction was performed by further reduction of the fatty tissue in the mons pubis and an additional surgical incision in the groin to resect excess skin (Fig. 4). Pathologic examination revealed no lipoblasts or atypical stromal cells but only mature adipocytes without nuclear atypia. Immunohistochemistry showed no expression of MDM2, CDK4, or p16 antigens. The patient's postoperative course was uneventful, and she was satisfied with her functional improvement, including walking and urination, as well as the esthetic outcome (Fig. 5).

\section{Discussion}

We described the case of a 46-year-old woman with bilateral giant lipomatosis in the vulvar region, which caused problems such as difficulty in walking and urination. MSL is so rare that the literature on this disease mainly comprises case reports and a small number of series. The incidence of MSL is approximately 1 in 25,000, and the male-to-female ratio is $15: 1$ to $30: 1[7,8]$. The disease mainly affects men between 30 and 60 years of age and develops within a few months or several years; not many women with MSL have been reported [9].

We retrospectively reviewed 50 articles on this subject published between 2000 and 2019 to investigate the clinical manifestation of MSL in women. Using PubMed and the search terms "multiple symmetric lipomatosis" and "Madelung disease," and excluding literature reviews without case reports, we found 106 cases in the English literature. As a result, 94 men (89\%) and 12 women (11\%) patients with MSL were identified, demonstrating a male-to-female ratio of $8: 1$. The mean age at presentation was $60.8 \pm 16.4$ years in women and $54.0 \pm 10.2$ years in men, while the mean age at onset was $54.6 \pm 10.5$ years in women and 46.6 \pm 11.4 years in men. Of the patients, $67 \%$ of women and $87 \%$ of men had a history of chronic alcohol consumption. MSL was characterized by diffuse symmetric accumulation of adipose tissue, most commonly around the neck region (85.8\%) followed by the shoulders $(35.8 \%)$, upper extremities $(22.6 \%)$, face (18.9\%), back (11.3\%), and chest (10.4\%) (Table 1). Notably, women with MSL had lipomatous masses in the upper extremities, lower extremities, and axillae significantly more frequently than did men. As for MSL affecting the perineal region, only 10 men and 1 woman have been reported worldwide (Table 2).

MSL is classified into 3 types according to fat mass distribution [19]. Type I is characterized by a neck distribution; lipomatous tissue is located around the neck and deltoid region, giving the patient a "horse collar" or "buffalo hump" appearance. Type
Fig. 1 Frontal (a) and lateral (b) views of a 46-year-old woman with a symmetric distribution of fat masses involving the vulvar region
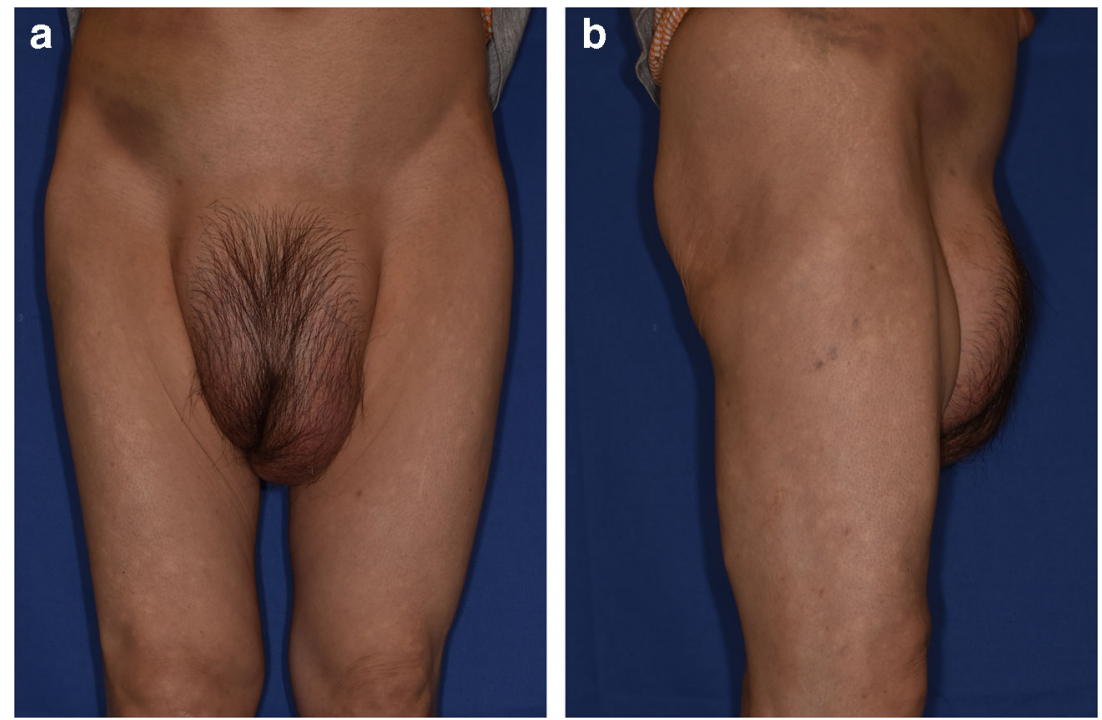
Fig. 2 Magnetic resonance imaging of the pubic area before surgery. T1-weighted axial images show high-signal intensity for fat deposition, which was diffuse and nonencapsulated in the labia majora (a) and extending around the rectum (b). c T2weighted coronal images show high-signal intensity without a specific sign of liposarcoma, such as linear or nodular foci of hyperintensities, on short tau inversion recovery sequences $(\mathbf{d})$
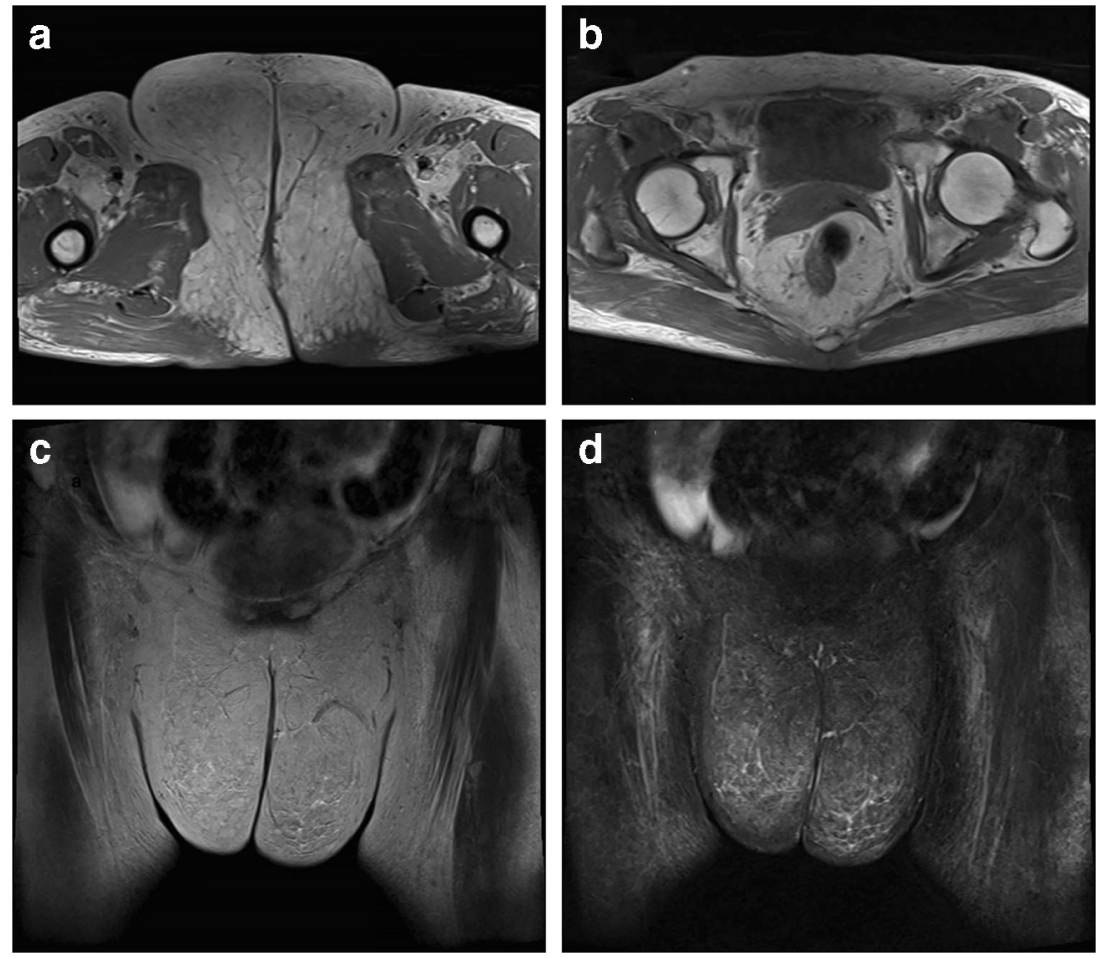
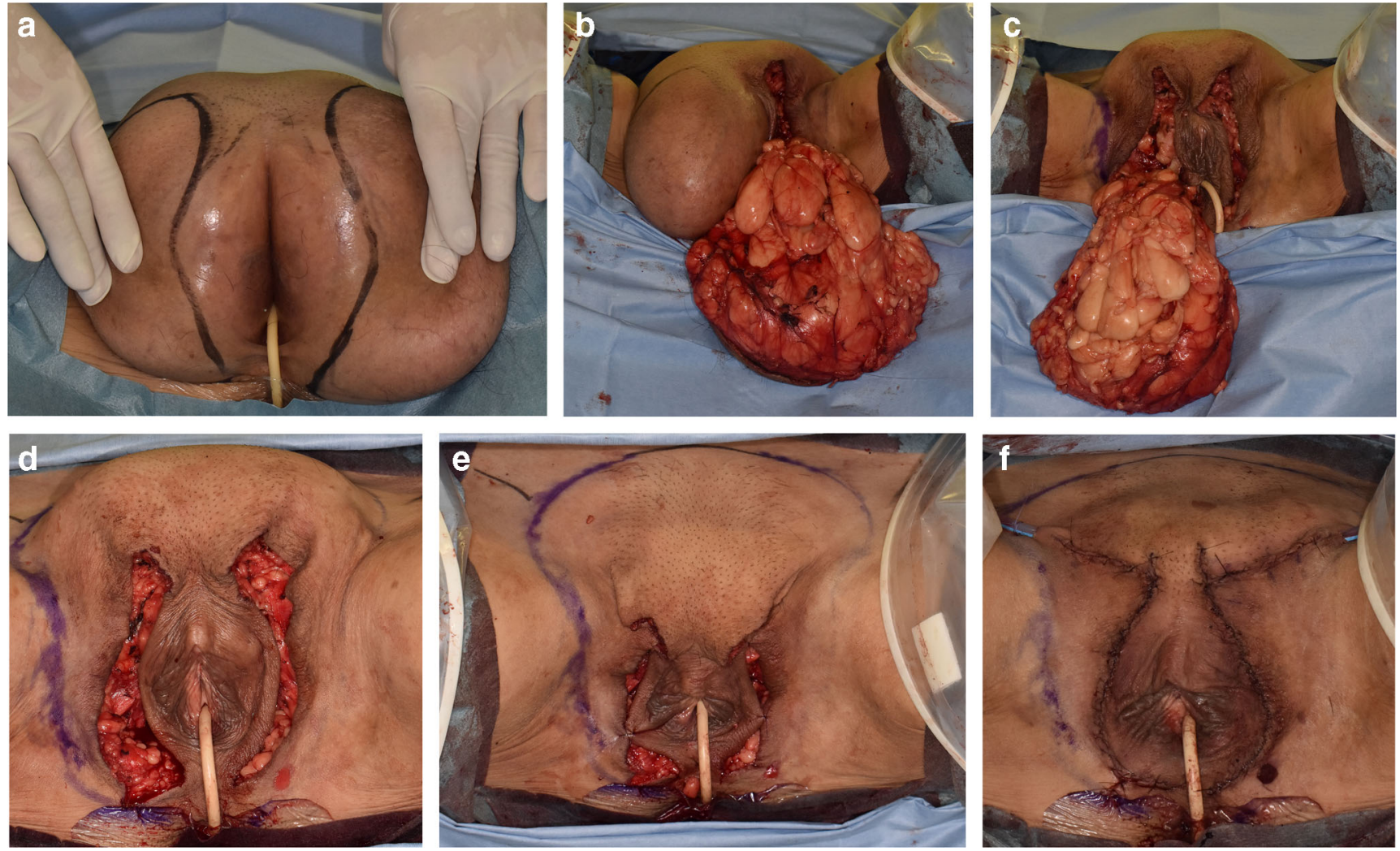

Fig. 3 Perioperative images of lipomatous tissue excision in the labia majora and mons pubis. a A crescent-shaped skin incision was made in the expanded skin of the labia majora. Inner lines were designed at least $4 \mathrm{~cm}$ apart from the labia minora. En bloc resection of skin and subcutaneous lipomatous tissue was performed in the left (b) and right (c) of the labia majora, taking care not to damage the vaginal and rectal wall. $\mathbf{d}$ The

swelling in the mons pubis still remained after the reduction of fatty tissue in the labia majora. e Further reduction of fatty tissue in the mons pubis was performed to an extent that did not impair the cutaneous blood flow. $\mathbf{f}$ The excess skin produced by the reduction of fatty tissue was excised after an additional surgical incision in the groin 
Fig. 4 Morphologic and pathologic images of the resected tissue. a, b Macroscopically, the excised tissue lacks encapsulation and the transition between lipomatous and normal tissue is poorly demarcated. $\mathbf{c}, \mathbf{d}$

Histologically, hematoxylin and eosin staining reveals mature adipocytes without cell nuclear atypia, whereas lipoblasts and atypical stromal cells were not found. Degenerating findings, such as fibrosis, myxoid change, mild infiltration of inflammatory cells, and appearance of macrophages, were also seen occasionally. The scale bar represents $2.5 \mathrm{~mm}$ in $\mathbf{c}$ and $250 \mu \mathrm{m}$ in $\mathbf{d}$
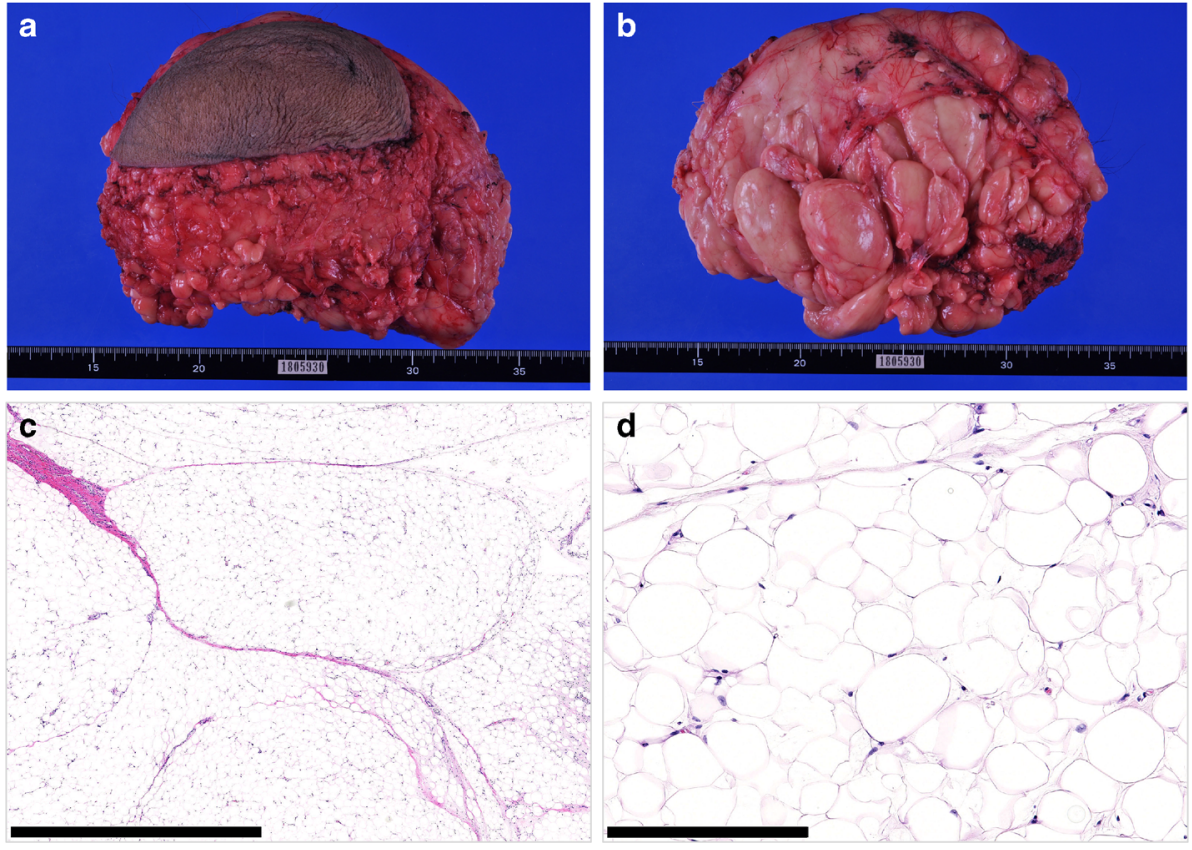

II is characterized by a pseudo-athletic appearance; lipomatous tissue is located throughout the body, including around the shoulders and upper extremities, and appears as simple obesity. Type III is characterized by a gynecoid appearance; lipomatous tissue is located in the lower body, including around the perineal region and lower extremities. However, some patients present with more than one type of distribution.

The diagnosis of MSL requires the presence of multiple nonencapsulated lipomatous tissues. Chronic alcohol consumption is a major risk factor, especially in men, and the disease may also be associated with certain internal diseases. Although MSL is generally easily diagnosed on physical examination, it can be clinically misdiagnosed as certain other diseases. CT and MRI are important to confirm the diagnosis by excluding other soft tissue tumors, such as liposarcoma [20]. CT can consistently show nonenhanced accumulation of fat tissue within subcutaneous tissue or the spaces between muscles. MRI is superior to CT in showing the structure of soft tissue, including excess nonencapsulated fat tissue.

The cause of MSL remains controversial but is suggested to be associated with alcohol abuse because $90 \%$ of patients with MSL have a history of chronic alcohol consumption.
Fig. 5 Frontal (a) and lateral (b) view of the postoperative results 6 months after lipectomy
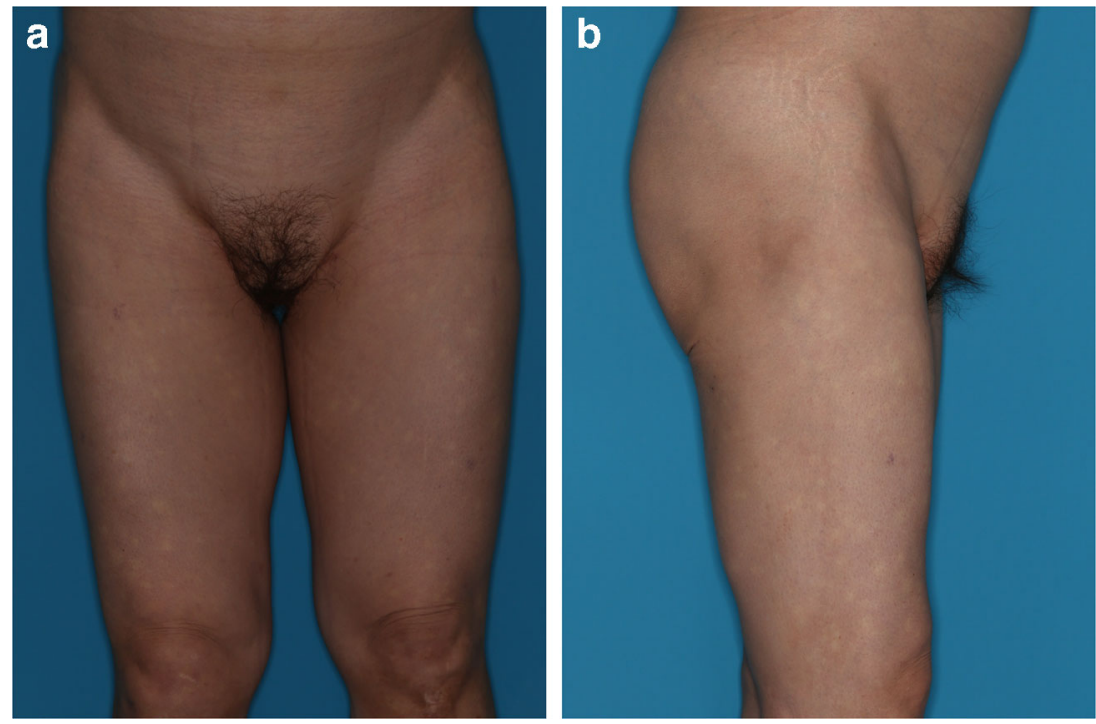
Table 1 Distribution of lipomatous masses in patients with MSL

\begin{tabular}{llll}
\hline Affected body areas & All patients, $n(\%)$ & Female, $n(\%)$ & Male, $n(\%)$ \\
\hline Neck region & $91(85.8 \%)$ & $9(75.0 \%)$ & $82(87.2 \%)$ \\
$\quad$ Posterior neck/occipital & $76(71.7 \%)$ & $7(58.3 \%)$ & $69(73.4 \%)$ \\
Anterior neck/submandibular & $56(52.8 \%)$ & $3(25.0 \%)$ & $53(56.4 \%)$ \\
Trapezium region/shoulders & $38(35.8 \%)$ & $4(33.3 \%)$ & $34(36.2 \%)$ \\
Upper extremities & $24(22.6 \%)$ & $7(58.3 \%)$ & $17(18.1 \%)$ \\
Face/periauricular & $20(18.9 \%)$ & $0(0.0 \%)$ & $20(21.3 \%)$ \\
Back & $12(11.3 \%)$ & $2(16.7 \%)$ & $10(10.6 \%)$ \\
Pectoral region/chest & $11(10.4 \%)$ & $0(0.0 \%)$ & $11(11.7 \%)$ \\
Perineal region & $7(6.6 \%)$ & $1(8.3 \%)$ & $6(6.4 \%)$ \\
Abdomen & $6(5.7 \%)$ & $1(8.3 \%)$ & $5(5.3 \%)$ \\
Lower extremities & $5(4.7 \%)$ & $2(16.7 \%)$ & $3(3.2 \%)$ \\
Axillae & $3(2.8 \%)$ & $2(16.7 \%)$ & $1(1.1 \%)$ \\
Buttocks & $1(0.9 \%)$ & $1(8.3 \%)$ & $0(0.0 \%)$ \\
\hline
\end{tabular}

There is no evidence that the disease is hereditary, and malignant transformation is rare [21]. Many hypotheses have been proposed in previous studies. One suggestion is that alcohol directly affects mitochondrial metabolism by causing oxidative aging of mitochondrial DNA [22]. However, some patients with MSL have no history of alcohol intake. In another clinical and histologic investigation, molecular-uncoupling protein $1 \mathrm{mRNA}$ and adipocyte fatty-acid protein- 2 immunohistochemistry findings suggested that the lipomatous tissue observed in MSL originates from brown adipocytes [23]. However, this hypothesis is also unsatisfactory because some patients with MSL have lipomatous tissue in regions where brown adipose tissue is considered absent, such as the pubic area.

Treatment for MSL includes both surgical and nonsurgical procedures [24]. As there is no definitive etiologically targeted treatment, all treatments are palliative, with the goal of function recovery and improved appearance. Open excision (lipectomy) may proceed to an important demolition in nononcologic diseases, but seems to be effective and safe because the vessels and nerves can be identified during the procedure. Liposuction is an easy method that can be performed safely under local anesthesia, but there is little clinical experience because it has not been widely used. A combined method of lipectomy and liposuction has also been reported [25]. When it is esthetically required to remove the skin as in the current case, lipectomy can provide a more desirable outcome compared with that with liposuction alone, which leaves sagging skin. Alternatively, liposuction of noncapsulated adipose tissue, with skin-only excision, would be an effective and less invasive option.

Intralipotherapy, a nonsurgical treatment, mainly limits growth of adipose masses and is not highly effective in reducing their volume. Alcohol withdrawal and weight loss may slow the enlargement of adipose masses and decrease the risk of recurrence after surgery, but cannot reverse the course of the disease. As for prognosis, patients with MSL who undergo lipectomy show a recurrence rate of $14 \%$ compared with $20 \%$ with
Table 2 List of MSL cases with involvement of the perineal region

\begin{tabular}{lllllll}
\hline Case no. & Authors & Age & Sex & Country & Alcoholism & Location \\
\hline 1 & Grolleau et al. (1997) [10] & 31 & M & France & Severe & Scrotum \\
2 & Soler et al. (1997) [11] & 30 & M & Spain & Severe & Scrotum, pubis \\
3 & Soler et al. (1997) [11] & 35 & M & Spain & Moderate & Scrotum \\
4 & Nounla et al. (2001) [12] & 14 & M & Germany & No & Perineum \\
5 & Poggi et al. (2006) [13] & 44 & M & Italy & Severe & Scrotum \\
6 & Tan et al. (2008) [6] & 20 & F & Turkey & No & Pubis \\
7 & Penna et al. (2012) [14] & 68 & M & Germany & Severe & Scrotum, pubis \\
8 & Alcantara et al. (2013)[15] & 54 & M & Brazil & Severe & Scrotum \\
9 & Nikolić et al. (2013) [16] & 51 & M & Serbia & Severe & Scrotum \\
10 & Turkan et al. (2015) [17] & 34 & M & Turkey & Unknown & Scrotum \\
11 & Ndhlovu et al. (2015) [18] & 58 & M & Germany & No & Perineum \\
12 & Costa et al. (2017) [5] & 53 & M & Portugal & Severe & Scrotum \\
13 & Present case & 46 & F & Japan & Severe & Labia majora \\
\hline
\end{tabular}


liposuction procedures. Specifically, ultrasound-assisted liposuction has shown a relapse rate of $50 \%$ [19].

In conclusion, we described a rare case of a middle-aged woman with MSL affecting the vulvar region. She had a history of chronic alcohol consumption and presented with giant fat masses in the labia majora, causing functional problems, including difficulty in walking and urination. The morphologic presentation of MSL in women is different from that in men, showing increased occurrence in the upper extremities, lower extremities, and axillae. Understanding of its characteristics could be helpful in avoiding a certain degree of the underdiagnosis of the disease in women. Clinical expression of MSL in the vulvar region is rare but cannot be overlooked due to the potential of causing serious sequelae, such as sexual dysfunction, urination disorder, and difficulty in walking. Lipectomy followed by episioperineoplasty is indicated to treat the condition and its sequelae, while providing a low risk of relapse and good esthetic outcome. MSL is a rare disease, especially in women, but it is important for plastic surgeons to keep such knowledge in mind when treating female patients with similar symptoms.

\section{Compliance with ethical standards}

Conflict of interest Maiko Kiyama, Koji Kanayama, Solji Roh, Yoshitsugu Hattori, Jun Oba, Takuya Iida, Tetsuo Ushiku, and Mutsumi Okazaki declare that they have no conflict of interest.

Ethical approval No ethical approval was necessary for this case report.

Informed consent Informed consent for publication was obtained from the patient in this case report.

Open Access This article is distributed under the terms of the Creative Commons Attribution 4.0 International License (http:// creativecommons.org/licenses/by/4.0/), which permits unrestricted use, distribution, and reproduction in any medium, provided you give appropriate credit to the original author(s) and the source, provide a link to the Creative Commons license, and indicate if changes were made.

\section{References}

1. Brodie BC (1846) Clinical lectures on surgery delivered at St. George's Hospital. Lea and Blanchard, Philadelphia, p 201

2. Madelung OW (1888) Über den Fetthals (diffuses Lipom des Halses). Arch Klein Chir 37:106-130

3. Launois PE, Bensaude R (1898) De l' adéno-lipomatose symétrique. Bull Mém Soc Med Hop (Paris) 1:298

4. Busetto L, Sträter D, Enzi G, Coin A, Sergi G, Inelmen EM, Pigozzo S (2003) Differential clinical expression of multiple symmetric lipomatosis in men and women. Int J Obes Relat Metab Disord 27:1419-1422

5. da Costa JN, Gomes T, Matias J (2017) Madelung disease affecting scrotal region. Ann Plast Surg 78:73-77

6. Tan O, Ergen D (2008) Madelung syndrome with pubic involvement. Dermatol Surg 34:811-814
7. Ruzicka T, Vieluf D, Landthaler M, Braun-Falco O (1987) Benign symmetric lipomatosis Launois-Bensaude. Report of ten cases and review of the literature. J Am Acad Dermatol 17:663-674

8. Zeitler H, Ulrich-Merzenich G, Richter DF, Vetter H, Walger P (2008) Multiple benign symmetric lipomatosis-a differential diagnosis of obesity. Is there a rationale for fibrate treatment? Obes Surg 18:1354-1356

9. Tadisina KK, Mlynek KS, Hwang LK, Riazi H, Papay FA, Zins JE (2015) Syndromic lipomatosis of the head and neck: a review of the literature. Aesthet Plast Surg 39:440-448

10. Grolleau JL, Soulie M, Dodart L, Costagliola M (1997) Scrotal location of Madelung's disease. Eur J Plast Surg 20:280-281

11. Soler R, Requejo I, Font-an FJ et al (1997) MR of laryngeal and scrotal involvement in multiple symmetrical lipomatosis. Eur Radiol 7:946-948

12. Nounla J, Rolle U, Gräfe G et al (2001) Benign symmetric lipomatosis with myelomeningocele in an adolescent: an uncommon association-case report. J Pediatr Surg 36:E13

13. Poggi G, Moro G, Teragni C, Delmonte A, Saini G, Bernardo G (2006) Scrotal involvement in Madelung disease: clinical, ultrasound and MR findings. Abdom Imaging 31:503-505

14. Penna V, Iblher N, Al-Jamali JM et al (2012) Rare locations of multiple symmetric lipomatosis (Madelung's disease). Eur J Plast Surg 35:557560

15. Alcantara KB, Reis Neto AJ, Ferreira AC, et al. (2013) Madelung's Disease With Large Involvement of the Scrotal Bag: Correlation of Two-Dimensional Ultrasonography With Full Body Tridimensional Computed Tomography. Ultrasound in Medicine \& Biology 39:S18

16. Nikolić ZS, Jeremić JV, Drčić LJ, Rakočević ZB, Tačević ZD, Jeremić KV, Stojnić JD (2013) Madelung disease: a rare case associated with gynaecomastia and scrotal involvement. J Plast Surg Hand Surg 47:415-418

17. Turkan S, Kalkan M, Șahin C (2015) Scrotal lipomatosis mimicking varicocele: a case report and review of the literature. Case Rep Urol 2015:695314

18. Ndhlovu M, Amr A, Schoeller T, Werdin F (2015) Localized lipomatosis of the perineum in a 58-year-old male patient - case report. Case Reports Plast Surg Hand Surg 2:31-33

19. Pinto CI, Carvalho PJ, Correia MM (2017) Madelung's disease: revision of 59 surgical cases. Aesthet Plast Surg 41:359-368

20. Zhang XY, Li NY, Xiao WL (2008) Madelung disease: manifestations of CT and MR imaging. Oral Surg Oral Med Oral Pathol Oral Radiol Endod 105:e57-e64

21. Tizian C, Berger A, Vykoupil KF (1983) Malignant degeneration in Madelung's disease (benign lipomatosis of the neck): case report. Br J Plast Surg 36:187-189

22. Gonzalez-Garcia R, Rodriguez-Campo FJ, Sastre-Perez J, et al (2004) Benign symmetric lipomatosis (Madelung's disease): case reports and current management. Aesthetic Plastic Surgery 28:108-12

23. Plummer C, Spring PJ, Marotta R, Chin J, Taylor G, Sharpe D, Athanasou NA, Thyagarajan D, Berkovic SF (2013) Multiple symmetrical lipomatosis-a mitochondrial disorder of brown fat. Mitochondrion 13:269-276

24. Constantinidis J, Steinhart H, Zenk J, Gassner H, Iro H (2003) Combined surgical lipectomy and liposuction in the treatment of benign symmetrical lipomatosis of the head and neck. Scand J Plast Reconstr Surg Hand Surg 37:90-96

25. Chen CY, Fang QQ, Wang XF et al (2018) Madelung's disease: lipectomy or liposuction? Biomed Res Int 2018:3975974

Publisher's note Springer Nature remains neutral with regard to jurisdictional claims in published maps and institutional affiliations. 